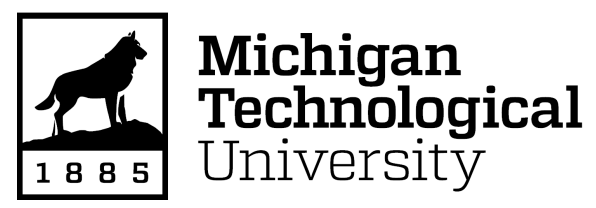

Michigan Technological University Digital Commons @ Michigan Tech

$6-2016$

\title{
An example of persistent microstructure in a long rain event
}

\author{
A. R. Jameson \\ RJH Scientific, Inc., El Cajon, California \\ M. L. Larsen \\ College of Charleston
}

A. B. Kostinski

Michigan Technological University

Follow this and additional works at: https://digitalcommons.mtu.edu/physics-fp

Part of the Physics Commons

\section{Recommended Citation}

Jameson, A. R., Larsen, M. L., \& Kostinski, A. B. (2016). An example of persistent microstructure in a long rain event. Journal of Hydrometeorology, 17(6), 1661-1673. http://dx.doi.org/10.1175/JHM-D-15-0180.1

Retrieved from: https://digitalcommons.mtu.edu/physics-fp/171

Follow this and additional works at: https://digitalcommons.mtu.edu/physics-fp

Part of the Physics Commons 


\title{
${ }^{\partial}$ An Example of Persistent Microstructure in a Long Rain Event
}

\author{
A. R. JAMESON \\ RJH Scientific, Inc., El Cajon, California \\ M. L. LARSEN \\ College of Charleston, Charleston, South Carolina \\ A. B. KostinsKi \\ Michigan Technological University, Houghton, Michigan \\ (Manuscript received 19 September 2015, in final form 8 March 2016)

\begin{abstract}
A 2D video disdrometer (2DVD) probe was used to gather detailed drop measurements over a 770-min rain event. Accumulated totals of the rainfall and of the number of drops for each square centimeter showed persistent, significant correlated structures across the approximately $11 \mathrm{~cm} \times 11 \mathrm{~cm}$ grid of the 2DVD. This is surprising because larger-scale studies suggest that the values in each square centimeter should be highly correlated with very little variation. Nevertheless, this correlation remains strikingly similar to what is observed at a coarser resolution, suggesting that it somehow scales with spatial resolution. However, because the correlation functions are not power laws, the origin of this scaling must be due to a factor other than fractal geometry. Analysis reveals that this occurs because of a filtering effect such that as the domain size (or resolution of a remote sensor) becomes finer, it is only the smaller wavelengths that contribute most to the variance so that the correlation function also scales. Consequently, correlated finescale structures can apparently occur even over $10 \mathrm{~cm}$. This fine structure was also found for the kinetic energy and impact power of the rain, important for understanding the initiation of soil erosion. The patterns in the integrated parameters appeared to arise almost exclusively from patterns in the total number of drops with patterns in the drop sizes playing an insignificant role. Therefore, in future studies of rain it is recommended that the total number of drops be retained as a crucial variable.
\end{abstract}

\section{Introduction}

Rainfall is the result of an intermittent, stochastic process occurring over a wide range of temporal and spatial scales. Consequently, it must be described statistically using the usual parameters of, for example, mean values, variances, and correlation functions. Perhaps the most frequently used parameter is the mean value averaged over time and/or space. However, all of these parameters are intimately related. Consequently, one should not really speak about the mean without

\section{¿ Denotes Open Access content.}

Corresponding author address: A. R. Jameson, 5625 N. 32nd St., Arlington, VA 22207-1560.

E-mail: arjatrjhsci@verizon.net mentioning a correlation length in time and space since it determines the accuracy and representativeness of that mean. This latter omission can be deceptively misleading.

A review of the literature often reveals an underlying assumption that the rain is statistically homogeneous, that is, its statistical measures are identical at all locations and times. This is often a necessary assumption in order to say anything meaningful using a set of observations. However, it may often be an incorrect assumption. That is, rain is frequently statistically heterogeneous so that its statistical properties depend on the times and locations of the measurements. At times this can have significant consequences because in those cases, for example, there may be no convergence to a constant either temporally or spatially no matter how extensive the observations. 
If we just focus on spatial variability, surely over small enough areas, such effects disappear in a uniform covering by the rain; after all, experience shows us that streets and car windshields become uniformly wet in only a brief time. Of course, this is misleading because "wetness" is an accumulative binary condition that, once satisfied, persists, while accumulated rainfall, for example, is an ongoing integral. Hence, it remains a valid question to ask, does rainfall spatial variability always disappear over some sufficiently small area or long enough period of observations even in statistically heterogeneous rain?

In this paper, we explore the answer by using the area of the 2D video disdrometer (2DVD) as a virtual network of 121 one-square-centimeter detectors. The 2DVD instrument is thoroughly described in Schönhuber et al. (2008). For this work, the relevant basic properties are that it creates two sheets of light having parallelogram sampling areas that are nearly square and that are separated by a small distance. When drops pass through, the blockages of the beams are measured by two chargecoupled device (CCD) cameras at orthogonal angles having a nominal resolution of $0.19 \mathrm{~mm}$ at sampling rates of $55 \mathrm{kHz}$. The dimensions, shapes, locations, and fall speeds of the drops are calculated and stored. Thus, the data can be subsequently categorized by time, location, and sizes as required for analyses.

The instrument is enclosed in a box out in an open field [see Fig. A3 in Jameson et al. (2015)] covered by low vegetation. An analysis on an older version of this instrument suggested that turbulence could affect some of the trajectories of the smaller drop sizes (Nešpor et al. 2000). The newer unit has since been redesigned in such a way that it minimizes these earlier effects. Even in the old version, however, the turbulence only extended $10-20 \mathrm{~cm}$ above the detector opening according to Nešpor et al. (2000) for a 3-5 $\mathrm{m} \mathrm{s}^{-1}$ ambient wind speed. Fortunately, the drop response times of the sizes most important to the rainfall rate $(0.6 \mathrm{~mm}$, the smallest size considered here, and larger diameters) would be much too long to feel any turbulence from the box encasing the measurement area. For drops of $0.6 \mathrm{~mm}$ diameter, Beard and Jameson (1983) show that the response time is a minimum of $0.26 \mathrm{~s}$, so that drops would have moved a minimum of $0.67 \mathrm{~m}$ or $0.4 \mathrm{~m}$ or more into the 2DVD detector before they could have even responded to any turbulence. Thus, the important drops would never have even felt any turbulence so that the observed spatial structures are likely unaffected.

In earlier work by Gires et al. (2015), the 2DVD was used as a single instrument to explore the fractal nature of rain. Our purpose here is entirely different. We use the combination of all of the 121 one-square-centimeter elements as a virtual spatial grid in order to map the 2D contours of variables and to study the statistical spatial structures of the rain using 2D correlation functions and radial spatial distribution functions. Before proceeding, however, we begin with two necessary definitions.

\section{Preliminary considerations}

Following Jameson and Larsen (2016a), let us consider a random variable $w$ as an element in a statistically homogeneous 2D field $W$. Let us also consider two locations at $\mathbf{r}$ and $\mathbf{r}+\Delta \mathbf{r}$. One can then define a correlation function between the $w$ observed at these two locations as

$$
\rho(\Delta \mathbf{r}, \theta)=\frac{\langle w(\mathbf{r}, \theta) w(\mathbf{r}+\Delta \mathbf{r}, \theta)\rangle-\mu^{2}}{\sigma^{2}},
$$

where $\sigma^{2}$ is the variance, $\mu$ is the mean value of $w$ over $W$ and the angle brackets denote an ensemble average over all locations separated radially and azimuthally by $\Delta \mathbf{r}$ and $\theta$, respectively. The angle brackets can also denote temporal averages when one uses direct calculations among instruments in a sparse network, as is done in Jameson et al. (2015). However, when there is a sufficiently dense grid of observations, the brackets are appropriately considered to represent an ensemble average. It is also worth noting that this quantity can be converted to the $2 \mathrm{D}$ pair correlation function (Kostinski and Jameson 2000; Shaw et al. 2002; Kostinski et al. 2006; Jameson et al. 2015) by multiplying by the spatial pair correlation function coefficient [SPCFC; SPCFC = $\left.\operatorname{var}(w) / \mu^{2}=(\mathrm{RD})^{2}\right]$, where $\mathrm{RD}$ is the relative dispersion and var is the variance.

One approach for calculating the 2D spatial correlation function is to use all the data simultaneously by first calculating the $2 \mathrm{D}$ power spectrum of the spatial distribution of $w$. That is, one first calculates $\mu$ and removes it from all the elements in $W$. The Fourier transform of these adjusted elements in this 2D matrix times its complex conjugate is then the variance spectrum, that is, the magnitude of the variance as a function of wavenumber (Blackman and Tukey 1975). If one then takes the inverse 2D Fourier transform of this variance spectrum, one derives the 2D correlation function by the Weiner-Khintchine theorem (Wiener 1930; Khintchine 1934). If $w$ were drop counts, for example, after normalization this correlation function is equivalent to $\rho(\Delta \mathbf{r}, \theta)$, or to the so-called 2D pair correlation function, if one multiplies by the SPCFC. On the other hand, if $w$ were the rainfall rate, then the result would simply be the 2D correlation function. As for any sample-by-sample calculation of a correlation function, the data must be 


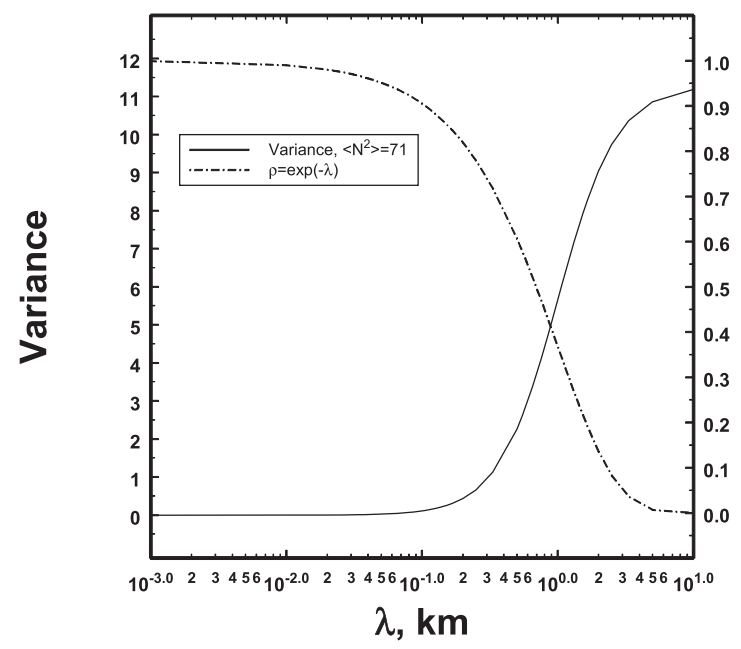

FIG. 1. An example of an exponential decaying correlation function having a $1 / e$ correlation length of $1 \mathrm{~km}$ and its associated variance spectrum as a function of wavelength $\lambda$. Small variance is associated with larger correlation and vice versa.

assumed or shown to be approximately statistically homogeneous. This is not always easy to demonstrate since the meteorology can introduce variable trends, although some methods for detecting this do exist (Anderson and Kostinski 2010). However, because we will only be using temporal averages of $\rho(\Delta \mathbf{r}, \theta)$, this is not an issue since trends and correlations can always be destroyed by reshuffling of the data, yet such reshuffling will not affect the average.

In addition, however, there is the so-called radial correlation function $\rho(\Delta r)$, which is just the azimuthal integration of $\rho(\Delta \mathbf{r}, \theta)$, that is,

$$
\rho(\Delta r)=\int_{\theta} \rho(\Delta \mathbf{r}, \theta) d \theta
$$

where $\theta$ is the azimuthal angle that, for this network, lies between 0 and $\pi / 2$ radians. In this work, $\rho(\Delta r)$ is calculated by converting the $2 \mathrm{D}$ correlation function in rectilinear coordinates into radius and azimuthal coordinates and then integrating at each $\Delta r$ over all the available angles. If one is concerned with drop counts, multiplication by the SPCFC converts the radial correlation function into the so-called radial distribution function of particle counts.

Finally, let us consider how a correlation function is related to the variance of a quantity. With regard to statistically homogeneous data, that is, data for which the statistical properties do not depend on location such as over the 2DVD area for sufficiently brief periods, this relation can be demonstrated by considering an exponentially decreasing $\rho(\Delta r)$ as in Fig. 1. The Fourier transform of $\rho(\Delta r)$ is the variance spectrum of the counts, that is, the magnitude of the variance as a function of wavenumber (inverse wavelength; Wiener 1930; Khintchine 1934; Blackman and Tukey 1975). Obviously, as the variance increases, $\rho(\Delta r)$ decreases and vice versa. This behavior has also been observed directly (Chudnovsky et al. 2013). It is also apparent that if the results in Fig. 1 were naively extrapolated to very small distances on the order of several centimeters, they would imply that $\rho(\Delta r)$ should be near unity with very small variance. Indeed, it is one reason for the prevalent idea that, over sufficiently small areas, fields like total rainfall smooth out, given enough time. However, such an extrapolation is misleading because Fig. 1 is based on a decreasing exponential correlation function having a characteristic $1 / e$ length of $1 \mathrm{~km}$. This implies measurements over a network having a dimension up to at least this scale in order to resolve a $1-\mathrm{km}$ wavelength. But what does that really tell us about observations on the much smaller scales of centimeters? Before addressing this explicitly in the next section, it is helpful first to show a few observations.

\section{Data and analyses}

\section{a. The $2 D$ spatial correlation functions}

The 2DVD is located at the historic Dixie Plantation near Hollywood, South Carolina; this property (owned by the College of Charleston Foundation) is used for a variety of ecological, educational, and research purposes. The site is located at $32^{\circ} 44^{\prime} 26^{\prime \prime} \mathrm{N}, 80^{\circ} 10^{\prime} 36^{\prime \prime} \mathrm{W}$. The primary data in these analyses occurred ahead of an approaching warm front with synoptic onshore flow from the east at a maximum of $5 \mathrm{~m} \mathrm{~s}^{-1}$ on 23 November 2014 beginning at 0815 EST and lasting continuously for slightly longer than $770 \mathrm{~min}$, with a period of more intense rain from 0 to $450 \mathrm{~min}$ followed by a period of lighter rain. During this period it was found that 10-min intervals yielded an average of almost 100 drops of diameter $D$ larger than $0.6 \mathrm{~mm}$ every square centimeter with many hundreds of such drops during the more intense rainfall.

One concern is the so-called "shadowing" effect of edges when a large tangential velocity of the wind passing over the instrument might distort the distribution of small drops near the upwind edge of the detector. For the data discussed here, the wind speeds were low (the synoptic wind speed was only $3 \mathrm{~m} \mathrm{~s}^{-1}$ on that day), and we only consider drops at least $0.6 \mathrm{~mm}$ in diameter having a fall speed sufficient so that they would not respond to such wind distortions as discussed further below. Moreover, just to be safe, we also eliminate edge counts. That is, drop positions ranged from 1 to $13 \mathrm{~cm}$, 


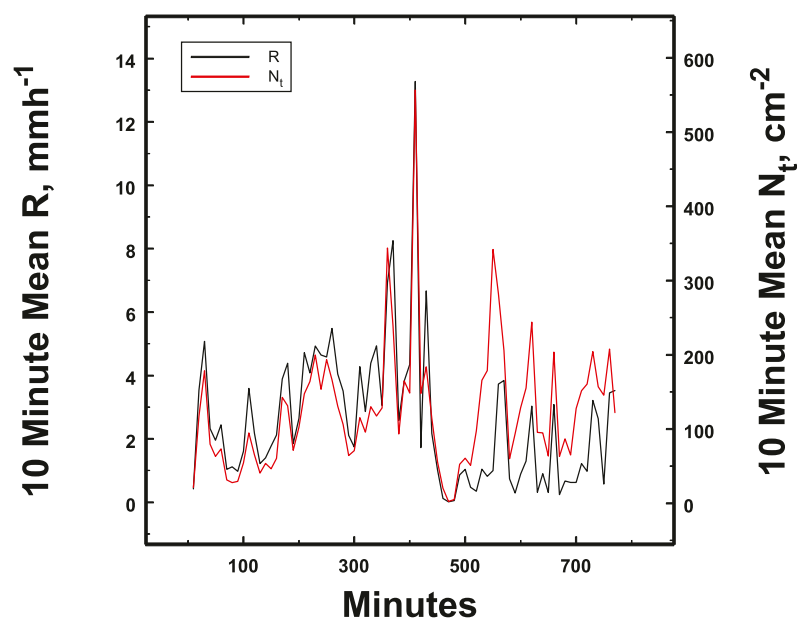

FIG. 2. Time series of the 2DVD 10-min average rainfall rate and the 10-min average number of drops per square centimeter during the 770-min rain.

but we only considered those that were within $2-12 \mathrm{~cm}$. Plots show that this is very effective in eliminating potential cutoff effects at edges.

Figure 2 is a plot of the average rainfall rate $R$ over the entire detector every $10 \mathrm{~min}$ as well as the average total number of drops $N_{t}$ per square centimeter having $D \geq 0.6 \mathrm{~mm}$. By and large, these data do not appear to be statistically stationary over the entire period. However, using the time series analyses approach of Anderson and Kostinski $(2010,2011)$, the data are actually nearly statistically stationary to within less than $1 \sigma$ of $\alpha=0$, so that these fluctuations are likely due to correlations. Consequently, over each individual 10-min period the observations are taken to be statistically stationary. Even though the clustering of drops in time and in space are different (Jameson et al. 2015), over the small area of the 2DVD, we also then assume that this stationarity implies that the observations are statistically homogeneous.

To investigate what happens spatially, the total drop counts and total rainfall are plotted in Figs. 3a and 3b, respectively. Significant spatial structures are clearly evident even when summing over the entire $770 \mathrm{~min}$. Here it is important to note that such features need not always be present. Furthermore, it is worth noting that on other days spatial features are still present, but they are located elsewhere on the grid. The spatial features are quite similar and have a $2 \mathrm{D}$ cross-correlation coefficient of 0.955 . Figures $3 \mathrm{c}$ and $3 \mathrm{~d}$ show what the fields should have looked like had the $x$ and $y$ position of each drop been completely random. Unlike Figs. 3a and 3b, Figs. $3 \mathrm{c}$ and $3 \mathrm{~d}$ do not show any particular structure, and they are completely different, having a $2 \mathrm{D}$ crosscorrelation coefficient of only 0.047 . Since structure is an indicator of correlation (Jameson and Larsen 2016a), these figures strongly suggest that even over a long time period and even over this small of a domain, some spatial correlation is retained, leading to much larger spatial differences than pure randomness would have generated. Moreover, there is apparently a physical connection between the total number of drops and the total rainfall that disappears when $x$ and $y$ are randomized. This will be discussed further later.

One response to such structure is "of course." After all, Jaffrain and Berne (2012) and Jameson et al. (2015) both show a high degree of correlation near null spatial separations. While this is true, extrapolation of these findings to near zero suggests that the correlation should have been nearly perfect so that every square centimeter should have had nearly the same value with small deviations. Obviously, this is not the case.

To see why this happens, it is first necessary to compute the $2 \mathrm{D}$ correlation function for each 10-min interval as described in the previous section. However, an accurate calculation of this quantity requires a good estimate of what the true mean value is during each 10-min spatial sample. Using minute-to-minute correlation functions, it was found that both $R$ and $N_{t}$ completely decorrelated in $30 \mathrm{~min}$. Consequently, we use the 10 -min values on either side of a particular 10-min interval to compute a least squared error-weighted estimate of the true mean for that interval and area of the 2DVD. These values are plotted for $N_{t}$ and $R$ in Figs. $4 \mathrm{a}$ and $4 \mathrm{~b}$, respectively. Here it is worth reminding the reader that spatial and temporal correlations are different (Jameson et al. 2015), so that a 30-min temporal decorrelation does not determine the spatial decorrelation length. To see this, one can imagine the hypothetical situation of a line or area of points having a steady particular spatial relation of values, but with the values at all the points varying temporally in unison so that they decorrelate in time without changing their spatial relation.

The averages of $\rho(\Delta \mathbf{r}, \theta)$ over 77 ten-minute intervals are plotted in Fig. 5. While there are rapid drops in the correlation within the first $2 \mathrm{~cm}$ in Figs. 5a and 5b, the values remain substantial $(>0.55)$ over the remainder of the area with some apparent anisotropy. On the other hand, for random $x$ and $y$ (Figs. 5c,d), the correlation drops to very small values by $2 \mathrm{~cm}$, not surprisingly, with values near zero over the remaining area. These results are reflected in the corresponding radial correlation functions as illustrated in Fig. 6. It is well known that the domain size affects the correlation function (e.g., Krajewski and Duffy 1988). Indeed, that is why the domain size is cut by two when showing the correlation functions. However, Fig. 6 shows that for random uncorrelated counts, the radial correlation drops to zero by one-quarter of the domain size, whereas the observed 

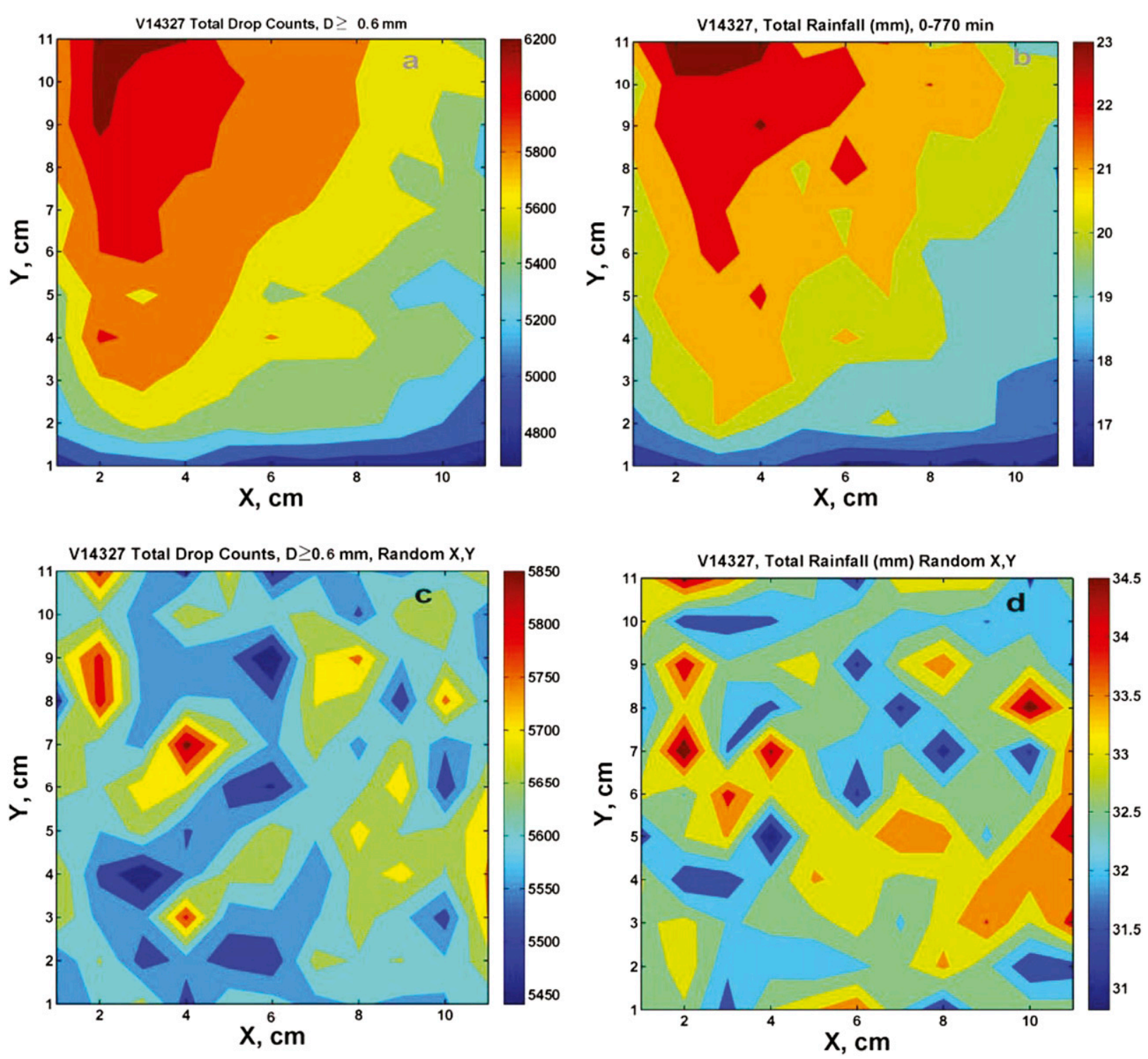

FIG. 3. Contour plots of the (a) total number of drops in $1-\mathrm{cm}^{2}$ boxes across the 2DVD viewing area over $770 \mathrm{~min}$, (b) total rainfall in each box, (c) total number of drops had the $x$ and $y$ positions of each drop been random, and (d) total rainfall had the drops positions been random. Obviously, there is much more structure in (a) and (b).

correlation functions continue quite strongly. Thus, the correlation appears realistic. Now another possible source of correlation is the inadequate estimation of the mean. First, the counts over each square centimeter are adequate since the relative error $(\sigma / \mu)$ that includes the effect of correlation is only $2 \%$. The spread in the counts is, therefore, $7 \sigma$. Furthermore, we described above (see Fig. 4) the method for estimating the network-wide mean values as a function of time. Hence, we are confident that our resulting correlation observations are meaningful.

We conclude, therefore, that the 2D correlations over such a small domain are real but certainly less than the values near unity implied by the extrapolation of results from observations at coarser resolutions. This also implies larger variances than might have been anticipated. Why is this?

The explanation is that there is a subtle scaling of the correlation function with spatial resolution. That is, as Fig. 1 illustrates at large wavelengths (coarse spatial resolution), the small wavelength variances do not contribute significantly and, therefore, the correlation function does not "feel" their presence. However, as the wavelength resolution decreases, the contribution of the variances at longer wavelengths decreases because of filtering (i.e., the changes introduced at larger scales 

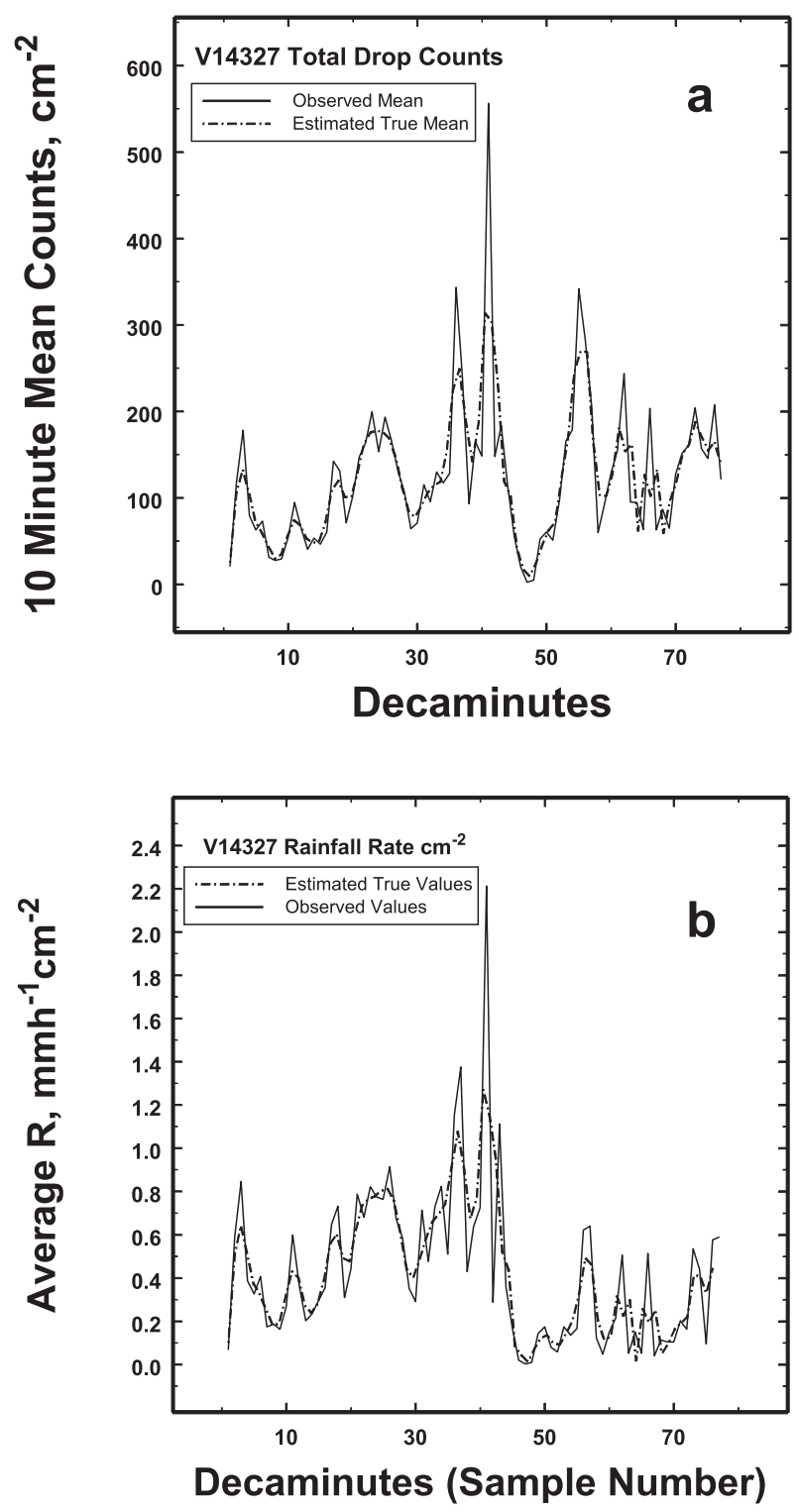

FIG. 4. Plots of the (a) estimates of the 10-min true mean of the total number of drops and (b) 10-min estimates of the $1-\mathrm{cm}^{2}$ true mean rainfall rate. Both are required for proper estimation of the $2 \mathrm{D}$ correlation function.

become part of the mean that is then removed when calculating the variance). Consequently, at finer spatial resolutions the variances associated with smaller wavelengths increase in relative importance so that the resulting correlation is reduced below what would have been expected by inappropriately extrapolating results at coarse resolution to finer resolutions. In other words, in that sense the correlation "scales" with resolution, but it is important to note that this scaling does not imply nor require fractal structure. The filtering of the variance with decreasing spatial resolution alone is apparently sufficient to scale the correlation function.

\section{b. Raindrop energy and power}

The existence of correlated structures in both the rainfall and total number of drops suggests that other such structures exist for other variables. One of the most important of these is the kinetic energy of the drops that plays a significant role in the process of soil erosion. Yet there is no information concerning the spatial structure of this variable over such small but important sizes of domains. That is, erosion is basically a small-scale phenomenon in which soil particles must first be dislodged by the kinetic energy (KE) of the larger drops and then carried away by the steadier supply of smaller drops (Kinnell 2005; Caracciolo et al. 2012).

Furthermore, as pointed out in a number of other contexts (Emanuel 2005; Wilson and Makris 2008), it is not just the kinetic energy $\left(\propto V^{2}\right)$ that is important but also the rate at which it is being delivered $\left(\propto V^{3}\right)$, where $V$ is the velocity of the air in hurricanes or the velocity of the rain drops. For the latter, this rate of delivery of kinetic energy has been referred to as rain power (Gabet and Dunne 2003), which is expressed as the time derivative of the rainfall rate. A more physically based definition is the drop impact power (IP) in which the kinetic energy of a drop of diameter $D$ is delivered in a characteristic time of $D / V$, where $V$ is the speed of the drop. Up to now, measurements of the kinetic energies and powers have been made at coarse resolutions in which the finescale structure is invisible. Yet, how this energy and power are distributed spatially over small areas in real rain is critical for a better characterization of the process of soil erosion (Gabet and Dunne 2003; Kinnell 2005).

To address this deficiency, we compute both the KE and IP for this rain event as illustrated in Fig. 7. Even over this small area and over $770 \mathrm{~min}$ of observations, there is considerable variability of roughly $\pm 30 \%$ around a mean value. These structures are reflected in the corresponding radial correlation functions (Fig. 8) for both KE and IP, which are essentially identical. However, what this illustrates is that microstructure on the centimeter scale exists and can significantly influence processes such as soil erosion. It is also likely that just as for the rainfall rate, the variabilities of both $\mathrm{KE}$ and IP increase with increasing spatial dimension (Jameson and Larsen 2016b).

Furthermore, studies using observations on larger scales suggest power-law relations between the rainfall rate and both KE (Brodie and Rosewell 2007) and the rain power (Gabet and Dunne 2003) having exponents in the range from near 1.1 to 1.4. In Fig. 9, the heavy dashed lines are linear fits over the observations. For both KE and IP, the correlations are 0.999 so that both 

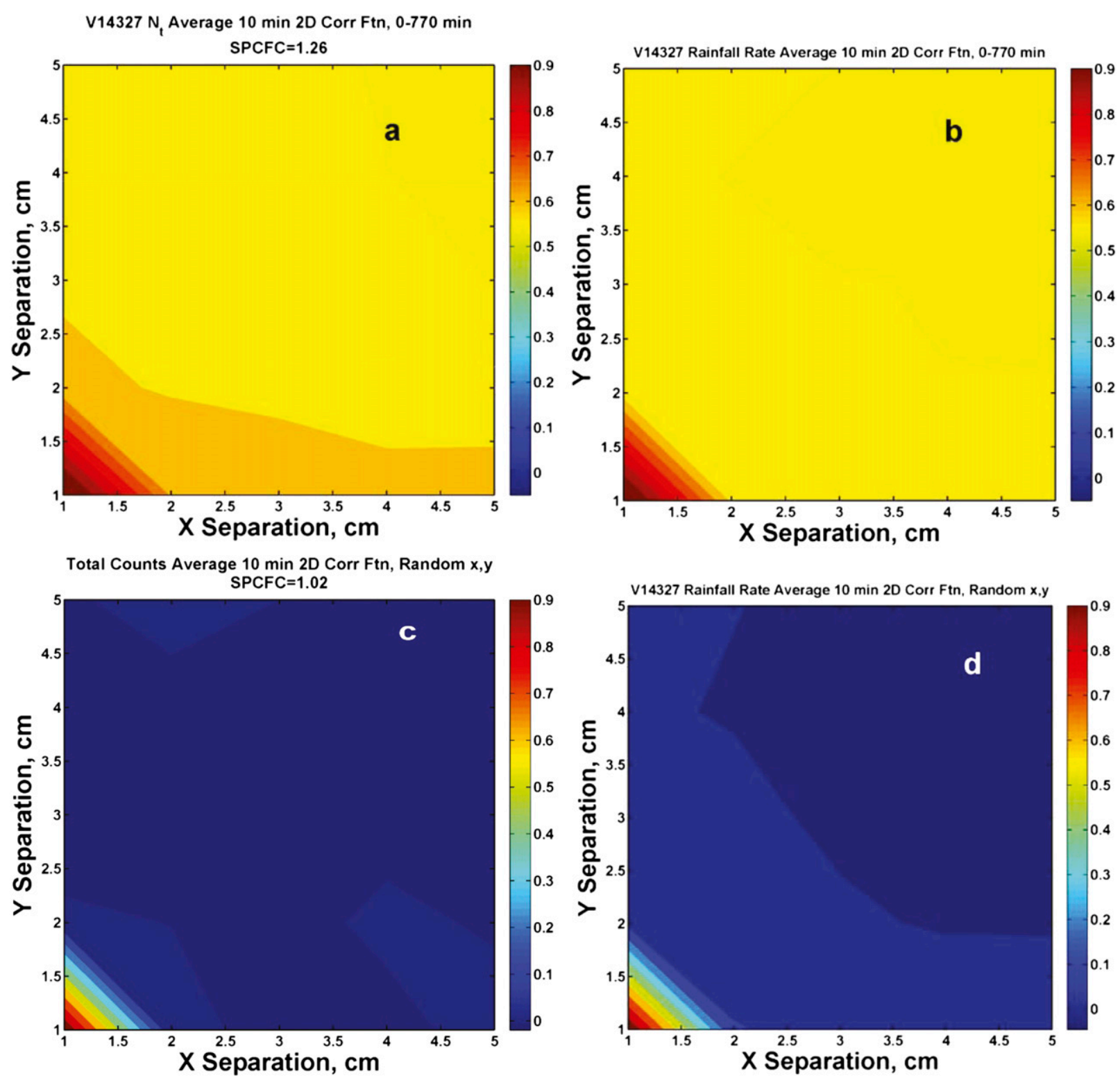

FIG. 5. Contour plots of the average of 10-min 2D correlation functions for the (a) total number of drops $(D \geq 0.6 \mathrm{~mm})$, (b) rainfall rate, (c) random drop position total number of drops, and (d) rainfall rate when the drop positions are random. A dramatic decrease in the spatial correlations caused by drop position randomization as compared to the observations is apparent.

variables can justifiably be considered to be linearly related to the total rainfall. However, these fits have nonzero intercepts at zero total rainfall. By forcing all the variables to intercept at zero, however, we then find power laws similar to those just mentioned. This is encouraging because it suggests such relations exist even over the scales most relevant to the earliest stages of soil erosion, but as Caracciolo et al. (2012) emphasize, it is the drop size distributions (DSDs) that are the bases for such relations. Hence, the variability evident in Fig. 9 must be a reflection of the variability of the DSD. However, the expression "drop size distribution" is somewhat ambiguous because a DSD consists of two components, namely, the total number of drops and the frequency distribution of the drop sizes $P(D) d D$, which expresses the probability of finding a drop with a diameter lying between $D$ and $D+d D$, so that the $\mathrm{DSD}=N_{t} \times P(D) d D$. With respect to the erosion capacity of rain, we find in the next section that the sizes of drops appear to be less important than the total number of drops for good physical reasons. 


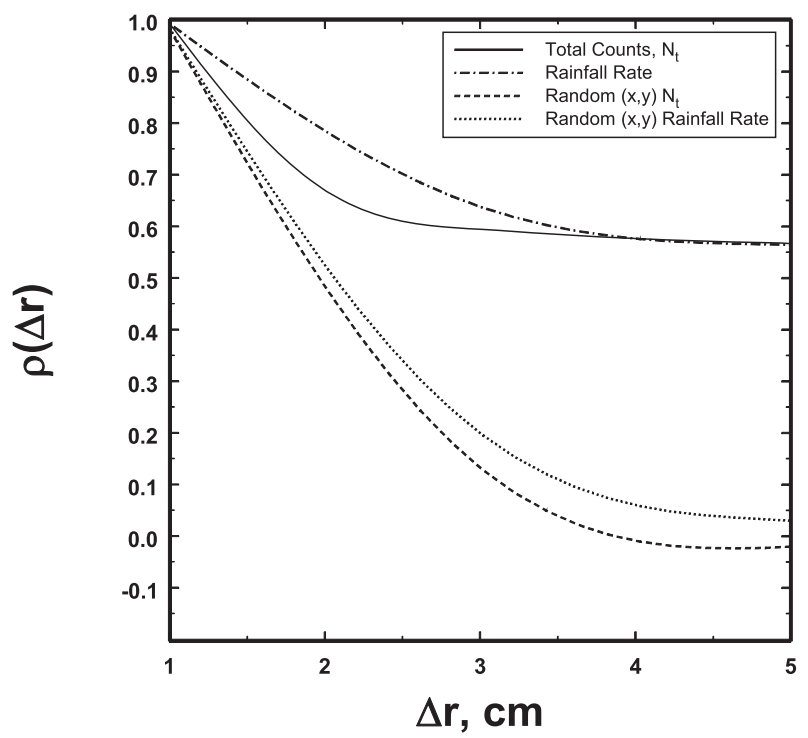

FIG. 6. The radial correlation functions corresponding to Fig. 5.

\section{c. On the 2DVD spatial variability of DSDs}

The sampling of a DSD depends on the underlying statistics. If the statistics are stationary in time or homogeneous in space, Jameson and Kostinski (2002a) found that it takes from tens of thousands to 100000 or more drops to define a DSD with sufficient accuracy to develop the correct relations among integral properties such as the rainfall rate and the radar reflectivity. This has also been confirmed in Larsen and O'Dell (2015, manuscript submitted to J. Atmos. Oceanic Technol.). If the statistics are nonstationary in time or heterogeneous in space, then the DSD keeps changing as more observations are combined without necessarily ever converging to a "steady" DSD (Jameson and Kostinski 2001). Here, we consider the entire set of data to see if they remain statistically homogeneous over the small observation area of the 2DVD. In that case, we have around an average (Fig. 3a) of 5500 drop counts per square centimeter, so that we can begin to explore the variability of the DSD at centimeter resolutions. While even this number of drops makes the definition of a complete DSD every square centimeter dubious, we can at least look at some integral properties beginning with the number-weighted mean diameter $\langle D\rangle$ (plotted in Fig. 10). This is chosen because, for exponential distributions, it is the inverse of the slope of the entire distribution (Kostinski and Jameson 1999). One is free to use other parameters, but this is sufficient for our purpose here, and more subtle differences are presented shortly.

Obviously, the variability in $\langle D\rangle$ is very small, amounting to only around $0.02 \mathrm{~mm}$ from the largest to
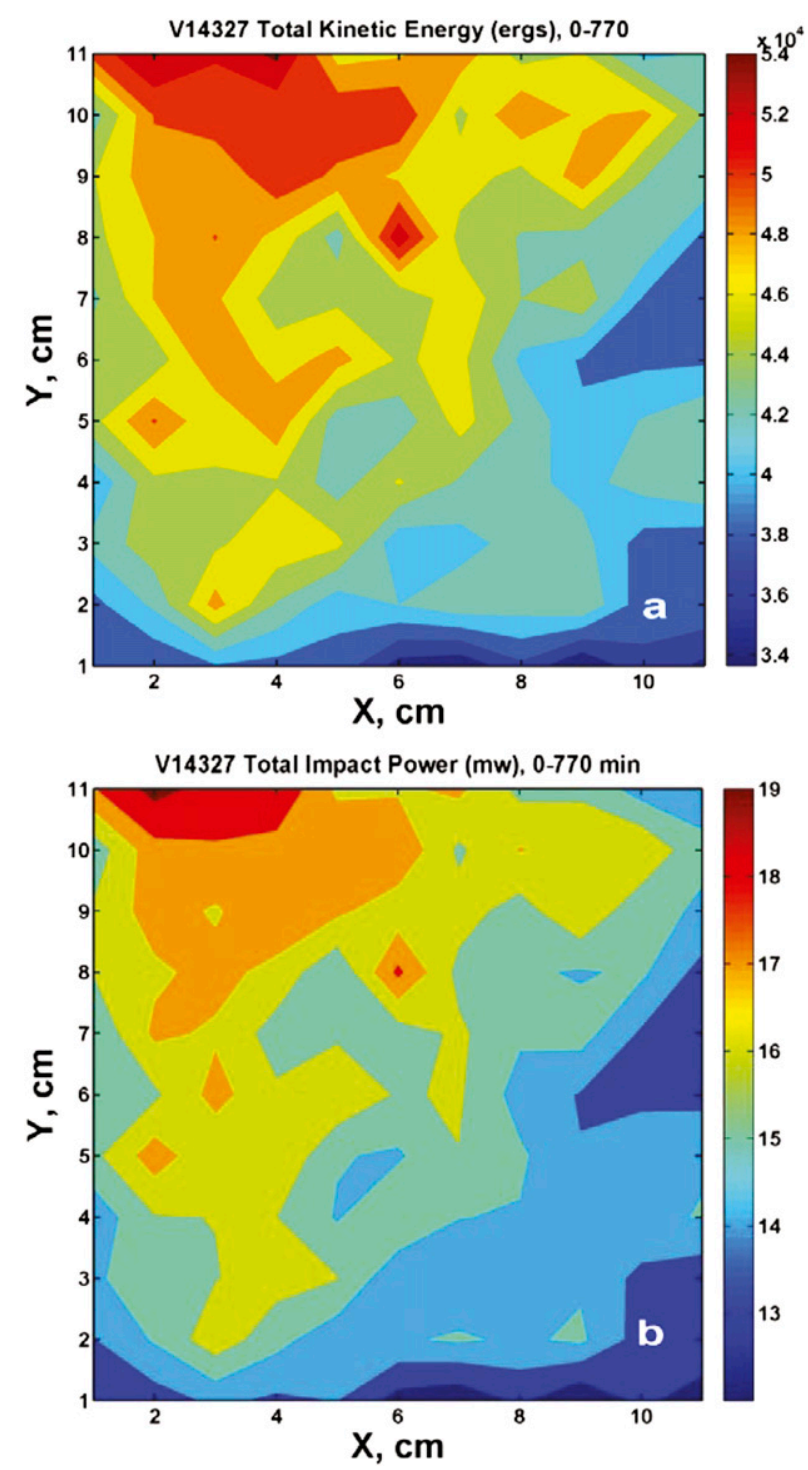

FIG. 7. Contour plots of (a) observed total KE of the rain and (b) IP showing persistent differences and structures on small scales.

the smallest values. This is the same as the resolution limitation of the drop size by the 2DVD, so such variations are not likely to be of any significance. So instead of just $\langle D\rangle$, let us instead consider the average spread of the different drop sizes for $D \geq 0.5 \mathrm{~mm}$ for each square centimeter. This is given by the $\mathrm{RD}\left(=\sigma_{D} /\langle D\rangle\right)$, where $\sigma_{D}$ and $\langle D\rangle$ are the standard deviations and mean sizes of all the drops, respectively, for each square centimeter. This is illustrated in Fig. 11.

The RD values are quite small. Had the distributions been exponential and extending over $[0, \infty)$, the relative dispersion would have been unity. One might suspect that truncation of exponential distribution at larger drop sizes might be responsible for shrinking the RD from 


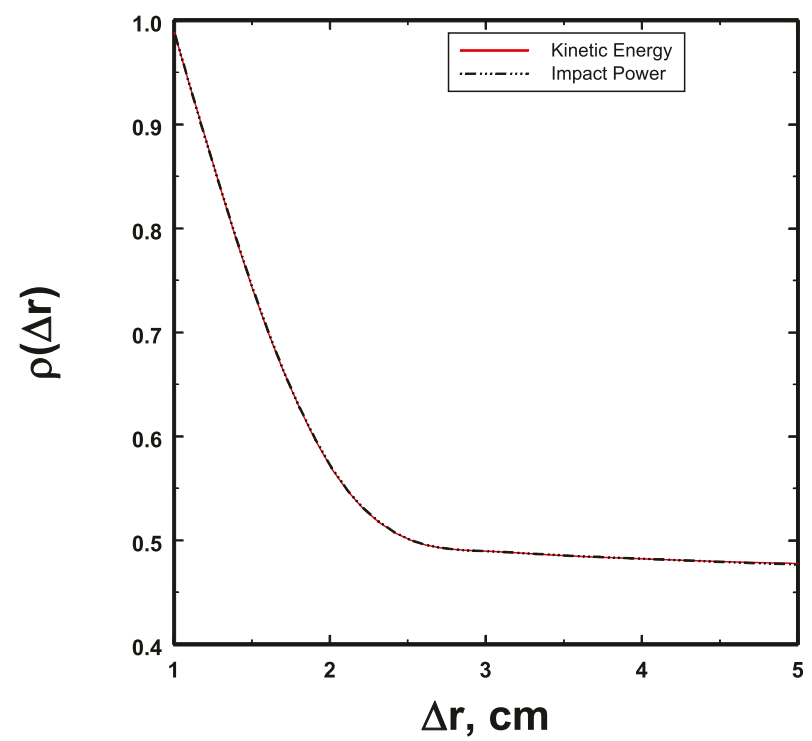

FIG. 8. The radial correlations functions corresponding for the KE and IP.

unity to the observed values. However, calculations show that for the minimum diameter of $0.6 \mathrm{~mm}$ used here, truncation at the larger drop sizes actually increases RD of an exponential to even greater values. Hence, with reasonable certainty we can at least conclude that on scales of $1 \mathrm{~cm}^{2}$, the DSDs are not exponential, but overall, the RD suggests that they are likely pretty similar, even though we cannot precisely determine their form at the $1-\mathrm{cm}^{2}$ scale.

However, if we combine several $1-\mathrm{cm}^{2}$ values together we will have enough drops to form a reasonable impression of a size distribution. To do this, we divide the area defined by $1 \leq X \leq 10 \mathrm{~cm}$ and $2 \leq Y \leq 11 \mathrm{~cm}$ into four sections each $5 \mathrm{~cm} \times 5 \mathrm{~cm}$ to give an average of over 130000 drops for estimating the DSD over each quadrant. The results are shown in Fig. 12.

The full DSDs $=N_{t} \times P(D) \Delta D$ are plotted in Fig. 12a, which clearly shows that they are quite similar. Moreover, if we perform a fit on all four $P(D) \Delta D$ (Fig. 12b), we find that the distributions are nearly identical and exponential. However, they are not perfectly identical, as Fig. 12c demonstrates. Here, we plot the accumulated absolute values of the differences between the mean distributions of drop sizes (PSD) in each quadrant from the overall average distribution $(\langle\mathrm{PSD}\rangle)$. While there are clear differences above about $2.5 \mathrm{~mm}$ diameter, the total deviations from the mean PSD over all the distributions are quite similar. Without much error, then, we conclude that over a sufficient number of squarecentimeter elements, the DSD do become essentially statistically homogeneous over the area of the 2DVD. For statistically homogeneous conditions (steady rain; Jameson and Kostinski 2002b; List 1988), Jameson and Kostinski (2002a) show that every integral property $Z$ can be expressed as a linear function of the total number

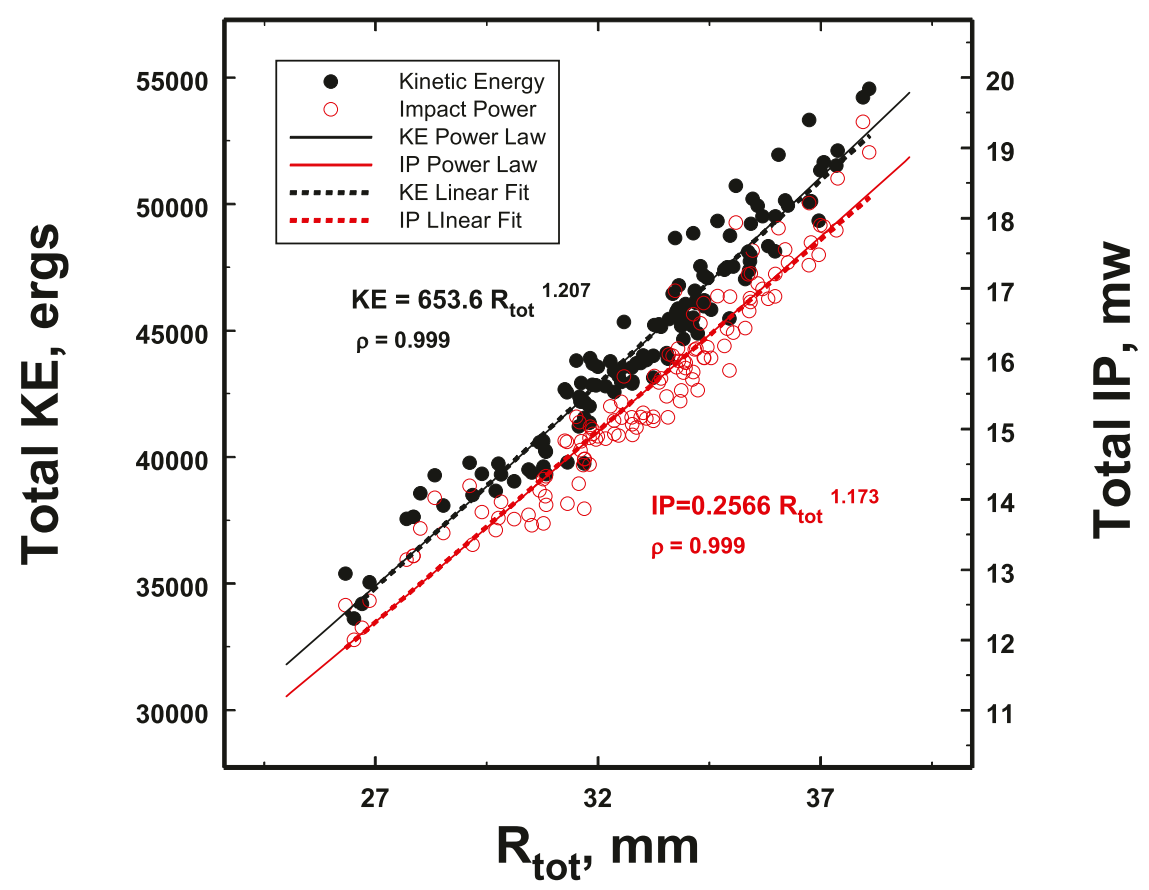

FIG. 9. Scatterplots and power-law fits of the total KE and total IP vs the total rainfall as discussed in the text. 


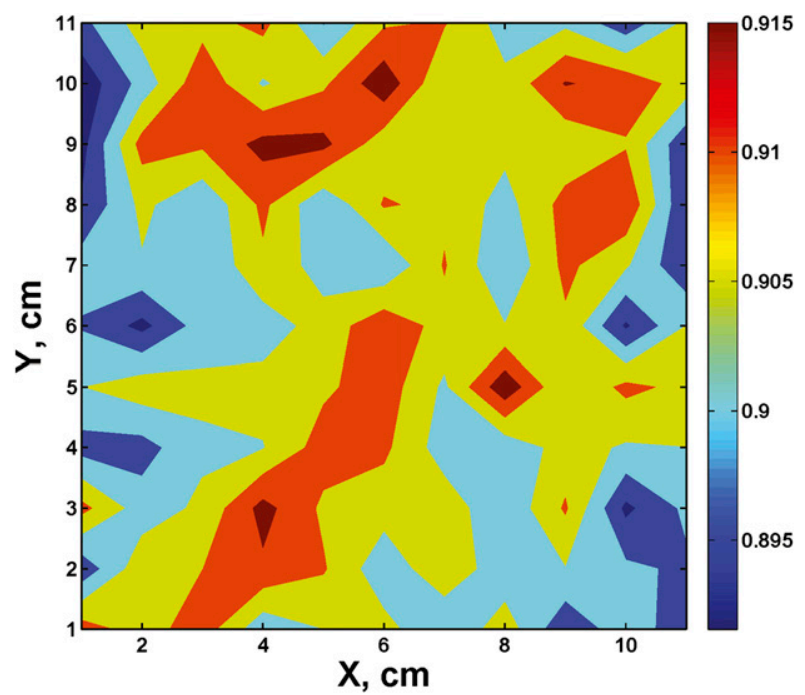

FIG. 10. The average number-weighted mean diameter for each $1 \mathrm{~cm}^{2}$. The variations are slight and on the order of the diameter resolution of the 2DVD.

of drops, that is, $Z=C \overline{D^{p}} N_{t}=C_{Z} N_{t}$ where $C_{Z}=C \overline{D^{p}}$, and where $D^{p}$ expresses the power dependence of $Z$ on $D$ and $C$ is a constant. In other words, all integral parameters are linearly related to $N_{t}$, and therefore they are all linearly related to each other. In particular, the rainfall rate is then linearly related to KE and IP, which are also then linearly related to each other. An example is provided in Fig. 13, where the $1-\mathrm{cm}^{2}$ values in Figs. $3 \mathrm{a}$ and $3 \mathrm{~b}$ are plotted in a scatter diagram. It is clear that the rainfall rate is linearly related to the total number of drops. Consequently, KE and IP should be linearly related to $R$. Even in statistically heterogeneous and nonstationary conditions, over a finite set of measurements there are still mean values of $D^{p}$ so that linearity still persists (Jameson et al. 2015) among integral properties but with greater scatter.

The fit of $R-N_{t}$ in Fig. 13 contains a constant that would yield negative $R$ at $N_{t}=0$. Obviously, this is nonsense brought about by the particular set of data used in determining the fit. One can, however, impose the additional constraint that $R=0$ when $N_{t}=0$. The imposition of this constraint leads to deviations from linearity in the relations between integrated parameters. This is not surprising because the constraint imposes a source of statistical heterogeneity not accounted for in the mean value of $D^{p}$. Thus, in Fig. 13, imposition of this constraint leads to a power-law relation (green). Similarly, power-law relations appear in Fig. 9 even though the variables are clearly linearly related over the range of the set of observations. The net conclusion, however, is that the variabilities evident in Figs. $3 b$ and 7 arise from the variability in $N_{t}$, not from the variability in $P(D)$. Thus, while $P(D)$ contributes to relationship among

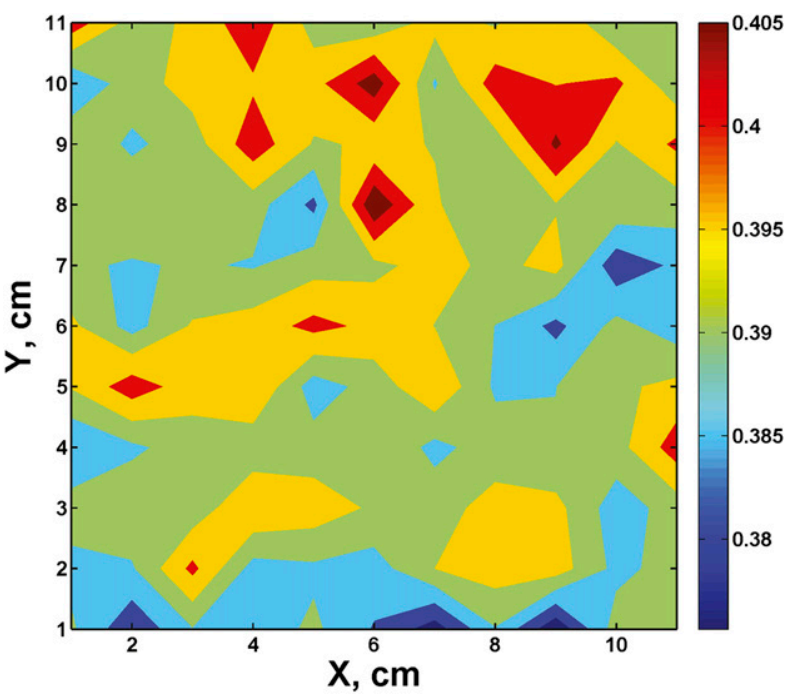

FIG. 11. A plot of the average RDs of the drop sizes indicating that the spread of the size distributions changes only slightly, as discussed in the text.

integrated variables, it is $N_{t}$ that drives their spatial variability.

This is similar to the findings when the characteristic length of a domain exceeds $1 \mathrm{~km}$ (Jameson and Larsen 2016b) and when it is only the dimension of a single Joss-Waldvogel disdrometer (Jameson 2015). On such small dimensions over reasonable times, the centimeter variability of $P(D)$ simply washes out, leaving $N_{t}$ as essentially the sole contributor to the variability of $R$. Thus, there appears to be a regime between around a few meters to $10 \mathrm{~m}$ out to distances approaching $1 \mathrm{~km}$ when $P(D)$ is an important factor driving the variability of the rainfall rates (Fig. 10; Jameson and Larsen 2016b); otherwise, $N_{t}$ is of the greatest importance and should be considered the origin of rainfall variability on most scales.

\section{Summary}

A 2DVD probe was used to gather detailed drop measurements over a 770-min rain event near Charleston, South Carolina, on 27 November 2014. Accumulated totals of the rainfall and of the number of drops for each square centimeter showed significant differences across the approximately $11 \mathrm{~cm} \times 11 \mathrm{~cm}$ grid of the 2DVD. This is surprising because larger-scale studies suggest that the values in each square centimeter should be highly correlated with very little variation. Moreover, these differences were not random but were spatially correlated, yielding structures. The spatial correlation, however, is strikingly similar to what is observed at coarser resolutions, suggesting that the spatial correlation 

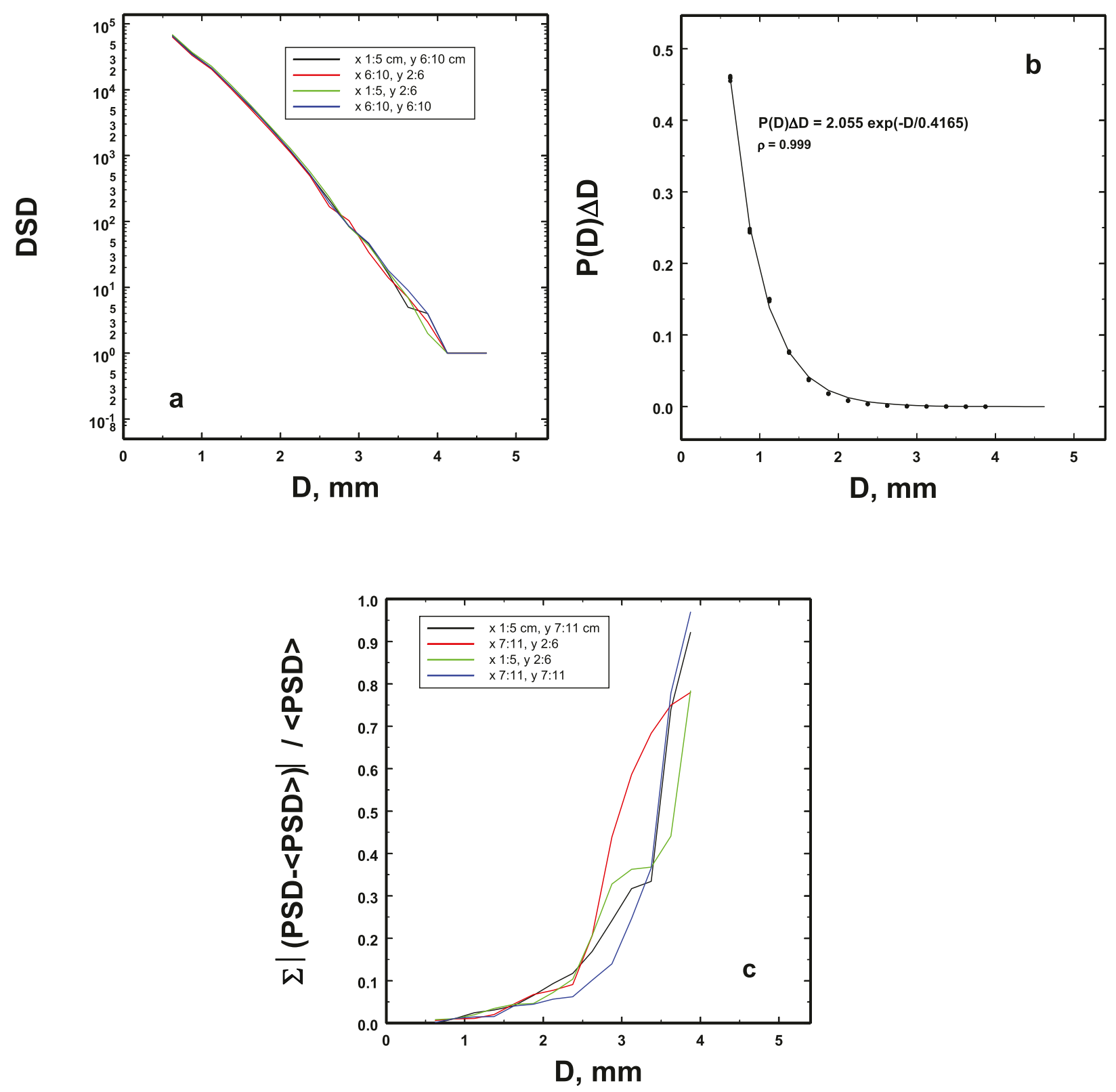

FIG. 12. Plots of the (a) DSD $=N_{t} \times P(D) \Delta D$ over four quadrants of the 2DVD sampling area and (b) fit to $P(D) \Delta D$ (=PSD) for the four quadrants, illustrating that the distributions are quite similar but not perfectly identical (c) when absolute deviations from the mean PSD are summed over the entire sample interval.

scales in some fashion with resolution. However, because the observed correlation functions are not power laws, the origin of this scaling must be due to a factor other than fractal geometry. It was concluded that this scaling with resolution occurs because of a filtering effect such that as a resolution becomes finer and finer, it is only the smaller scales that contribute most to the variance. These determine the local correlation function. However, as the resolution becomes coarser and coarser, the finescale contributions to the variance become negligible, so that it is the larger scales that then determine the correlation function. The important point, however, is that one cannot extrapolate coarser-resolution observations to infer the absence of structures on finer scales. As we see in this study, there is structure even on $10-\mathrm{cm}$ scales over long times.

This fine structure was also found for the kinetic energy (KE) of the rain. An impact power was then defined for each drop as the KE of the drop delivered in a characteristic time of $D / V$, where $D$ is the drop diameter 


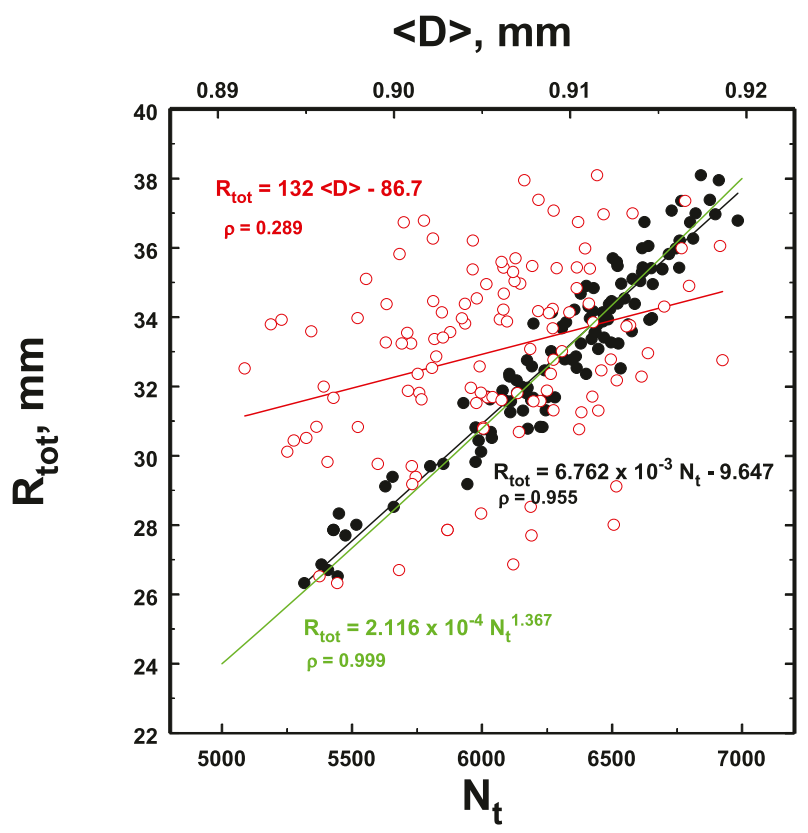

FIG. 13. Scatterplots of the total rainfall $R_{\mathrm{tot}}$ vs the total drop counts and number-weighted mean drop size over each square centimeter. While $R_{\text {tot }}$ is only weakly correlated to $\langle D\rangle$ (red), it is strongly correlated to $N_{t}$ (black) over each square centimeter, just as found in previous studies over much larger domains. The power-law fit (green) satisfies the requirement that $R_{\text {tot }}$ be zero when $N_{t}$ is zero.

and $V$ is the fall speed of the drop. Summation over all the drops then yields the total rain impact power (IP). Both of these quantities are important to initiating soil erosion (Brodie and Rosewell 2007; Kinnell 2005), and both showed structure and significant correlation across the sampling area of the 2DVD. This suggests that it is not sufficient just to use only coarse-resolution data when studying soil erosion, particularly since it is initiated at the soil particle scale.

We also considered variations in the drop size distribution (DSD). The DSD is given by $N_{t} \times P(D) \Delta D$, where $N_{t}$ is the total number of drops and $P(D) \Delta D$ is the frequency distribution of the drop sizes. It was found over the $770 \mathrm{~min}$ that net spatial patterns of $P(D) \Delta D$ were small, occurring mostly at larger drop sizes. Consequently, the net patterns in the various parameters such as the rainfall, KE, and IP can be attributed predominately to the net spatial patterns of $N_{t}$ over the period of observations and not so much to the patterns in $P(D) \Delta D$. Therefore, in future studies it is important to keep careful track of the total number of drops.

Finally, if the rain is steady (Jameson and Kostinski 2002b), significant spatial structures need not appear, depending on the amount of correlation. However, when the rain shows significant fluctuations in time, as often occurs in convective situations, spatial structures such as the one example described here are likely present.
Acknowledgments. This work was supported for A.J. by the National Science Foundation (NSF) under Grants AGS130087 and AGS1532423, as well as by the United States Social Security Administration. Support for M.L. came from the National Science Foundation under Grants AGS-1230240 and AGS1532977. Support for A.K. came from NSF Grant AGS-111916.

\section{REFERENCES}

Anderson, A., and A. Kostinski, 2010: Reversible record breaking and variability: Temperature distributions across the globe. J. Appl. Meteor. Climatol., 49, 1681-1691, doi:10.1175/ 2010JAMC2407.1.

— and - 2011: Evolution and distribution of record-breaking High and low monthly mean temperatures. J. Appl. Meteor. Climatol., 50, 1859-1871, doi:10.1175/JAMC-D-10-05025.1.

Beard, K. V., and A. R. Jameson, 1983: Raindrop canting. J. Atmos. Sci., 40, 448-454, doi:10.1175/1520-0469(1983)040<0448: $\mathrm{RC}>2.0 . \mathrm{CO} ; 2$.

Blackman, R. B., and J. W. Tukey, 1975: The Measurement of Power Spectra from the Point of View of Communications Engineering. Dover Publications, $190 \mathrm{pp}$.

Brodie, I., and C. Rosewell, 2007: Theoretical relationships between rainfall intensity and kinetic energy variants associated with stormwater particle washoff. J. Hydrol., 340, 40-47, doi:10.1016/j.jhydrol.2007.03.019.

Caracciolo, C., M. Napoli, F. Porcù, F. Prodi, S. Dietrich, C. Zanchi, and S. Orlandini, 2012: Raindrop size distribution and soil erosion. J. Irrig. Drain. Eng., 138, 461-469, doi:10.1061/(ASCE)IR.1943-4774.0000412.

Chudnovsky, A. A., A. Kostinski, A. Lyapustin, and P. Koutrakis, 2013: Spatial scales of pollution from variable resolution satellite imaging. Environ. Pollut., 172, 131-138, doi:10.1016/ j.envpol.2012.08.016.

Emanuel, K., 2005: Increasing destructiveness of tropical cyclones over the past 30 years. Nature, 436, 686-688, doi:10.1038/ nature 03906 .

Gabet, E. J., and T. Dunne, 2003: Sediment detachment by rain power. Water Resour. Res., 39, 1002, doi:10.1029/2001WR000656.

Gires, A., I. Tchiguirinskaia, D. Schertzer, and A. Berne, 2015: 2DVD data revisited: Multifractal insights into cuts of the spatiotemporal rainfall process. J. Hydrometeor., 16, 548-562, doi:10.1175/JHM-D-14-0127.1.

Jaffrain, J., and A. Berne, 2012: Quantification of the small-scale spatial structure of the raindrop size distribution from a network of disdrometers. J. Appl. Meteor. Climatol., 51, 941-953, doi:10.1175/JAMC-D-11-0136.1.

Jameson, A. R., 2015: A Bayesian method for upsizing single disdrometer drop size counts for rain physics studies and areal applications. IEEE Trans. Geosci. Remote Sens., 53, 335-343, doi:10.1109/TGRS.2014.2322092.

— and A. B. Kostinski, 2001: What is a raindrop size distribution? Bull. Amer. Meteor. Soc., 82, 1169-1177, doi:10.1175/ 1520-0477(2001)082<1169:WIARSD>2.3.CO;2.

$\longrightarrow$, and - 2002a: Spurious power-law relations among rainfall and radar parameters. Quart. J. Roy. Meteor. Soc., 128, 2045-2058, doi:10.1256/003590002320603520.

, and - 2002b: When is rain steady? J. Appl. Meteor., 41, 83-90, doi:10.1175/1520-0450(2002)041<0083:WIRS >2.0.CO;2.

, and M. L. Larsen, 2016a: Estimates of the statistical twodimensional spatial structure in rain over a small network 
of disdrometers. Meteor. Atmos. Phys., doi:10.1007/ s00703-016-0438-0, in press.

$\longrightarrow$, and — 2016b: The variability of the rainfall rate as a function of area. J. Geophys. Res. Atmos., 121, 746-758, doi:10.1002/2015JD024126.

,-- , and A. B. Kostinski, 2015: Disdrometer network observations of finescale spatial-temporal clustering in rain. J. Atmos. Sci., 72, 1648-1666, doi:10.1175/JAS-D-14-0136.1.

Khintchine, A., 1934: Korrelationstheorie der stationaren stochastischen Prozesse. Math. Ann., 109, 604-615, doi:10.1007/ BF01449156.

Kinnell, P. I. A., 2005: Raindrop-impact-induced erosion processes and prediction: A review. Hydrol. Processes, 19, 2815-2844, doi:10.1002/hyp.5788.

Kostinski, A. B., and A. R. Jameson, 1999: Fluctuation properties of precipitation. Part III: On the ubiquity and emergence of the exponential drop size spectra. J. Atmos. Sci., 56, 111-121, doi:10.1175/1520-0469(1999)056<0111:FPOPPI >2.0.CO;2.

$\longrightarrow$, and _ 2000: On the spatial distribution of cloud particles. J. Atmos. Sci., 57, 901-915, doi:10.1175/1520-0469(2000)057<0901: OTSDOC $>2.0 . \mathrm{CO} ; 2$.

_- M. L. Larsen, and A. R. Jameson, 2006: The texture of rain: Exploring stochastic micro-structure at small scales. J. Hydrol., 328, 38-45, doi:10.1016/j.jhydrol.2005.11.035.
Krajewski, W. F., and C. J. Duffy, 1988: Estimation of correlation structure for a homogeneous isotropic random field: A simulation study. Comput. Geosci., 14, 113-122, doi:10.1016/ 0098-3004(88)90055-6.

List, R., 1988: A linear radar reflectivity-rainrate relationship for steady tropical rain. J. Atmos. Sci., 45, 3564-3572, doi:10.1175/ 1520-0469(1988)045<3564:ALRRRF>2.0.CO;2.

Nešpor, V., W. F. Krajewski, and A. Kruger, 2000: Wind-induced error of raindrop size distribution measurement using a two-dimensional video disdrometer. J. Atmos. Oceanic Technol., 17, 1483-1492, doi:10.1175/1520-0426(2000)017<1483:WIEORS > 2.0.CO;2.

Schönhuber, M., G. Lammer, and W. Randeu, 2008: The 2D-videodistrometer. Precipitation: Advances in Measurement, Estimation and Prediction, S. Michaelides, Ed., Springer, 3-31, doi:10.1007/978-3-540-77655-0_1.

Shaw, R. A., A. B. Kostinski, and M. L. Larsen, 2002: Towards quantifying droplet clustering in clouds. Quart. J. Roy. Meteor. Soc., 128, 1043-1057, doi:10.1256/003590002320373193.

Wiener, N., 1930: Generalized harmonic analysis. Acta Math., 55, 117-258, doi:10.1007/BF02546511.

Wilson, J. D., and N. C. Makris, 2008: Quantifying hurricane destructive power, wind speed, and air-sea material exchange with natural undersea sound. Geophys. Res. Lett., 35, L10603, doi:10.1029/2008GL033200. 